\title{
GPS 技术在工程测量中的有效应用
}

楼宇鹏

新昌县华宇测绘有限公司

DOI:10.32629/gmsm.v2i4.214

[摘要] 社会经济的发展与进步,促使科学技术得到了很大程度的发展,特别是我国的工程测量中,GPS 技术得到了广泛应用。 本文就将对 GPS 技术在工程测量中的应用进行探讨, 以供参考。

[关键词] GPS 技术; 工程测量; 应用

近几十年来, 我国信息技术的发展尤为迅速, GPS 的应 用也变得越来越普遍。起初 GPS 主要被应用在定位上, 不过 随着技术性能的完善, 其应用范围逐渐拓展。尤其是该技术 在工程测量中的应用, 不仅降低了工程测量难度, 保证了测 量工作的精准性, 也减少了测量成本的损耗, 提高了工程测 量效率。为此加强 GPS 技术在工程测量中的应用研究尤为 关键。

\section{GPS 技术与工程测量}

GPS 是全球定位系统的简称, 该系统主要是通过卫星及 定位系统的应用实现对各空间区域特定物体坐标的定位和 控制, 帮助相关领域人员及时、高效的获取所需数据信息, 提升工作效率。随着科学技术的发展, 全球定位系统逐渐被 应用到工程测量中, 并取得了显著成效。通过该技术的应用 实现了不同地质、不同气候条件下, 被测物体的实时监控, 明确被测物体所处环境, 及周边特征。目前全球定位系统在 导航、均是、野外考察、土地测量等领域中贡献较大。

工程测量是建筑项目实施中涉及的所有工程测绘活动 的总称。现阶段各领域项目开展前, 都需要进行准确的工程 测量活动, 以此来获取精准的测量数据, 为工程项目建设方 案的制定提供依据。由于测量对象的不同, 工程测量的种类 也不尽相同, 如建筑工程测量、水利工程测量、矿区工程测 量、交通基础设施测量以及均是测量等。不管是哪种测量工 作, 其核心目标都是通过测量数据的掌握来了解项目开展中 可能出现的问题, 并制定合理的解决方案, 从而降低项目风 险, 保证企业的经济效益。而全球定位技术在建筑测量中的 应用, 能够实现全天候测量工作, 以此加强测量数据的精准 性, 优化测量水平。

\section{GPS 测量技术的优势}

2.1 定位快速、高效

应用全球定位技术, 工程测量可以开启实时定位模式, 实现对测量点的动态化管理。在实际操作中, 只需建立相应 的流动观测站, 并确保其运行状态稳定, 即可通过全球定位 技术来对每个观测站中的观测数据进行实时收集和处理, 这 大大缩短了测量时间, 提高了测量效率。

\section{2 全天候观测}

通过全球定位测量技术的应用, 可以在不同时间、不同
地点开展连续观测工作, 降低了传统测量中气候因素对测量 结果的影响, 加强了测量数据的准确性。同时, 通过该技术的 应用, 还可以实现被测点的快速定位以及信息获取, 提高了 测量工作的效率。

\section{3 测量精准度高}

传统工程测量主要是通过人工测量方式做到的, 这使得 测量工作会受到很多因素的影响而出现误差。但是全球定位 测量技术, 是通过卫星技术对被测物体进行准确把控来完成 测量工作的, 其可降低不良因素的影响, 增强测量结果的准 确性。据相关数据统计, 采用 GPS 技术开展的工程测量, 其静 态定位距离可精确到毫米左右, 动态测量中误差值可精确到 厘米以下。

\section{4 操作简单}

GPS 测量技术在应用过程中需要通过接收机来完成信号 收集和传输。随着技术的快速发展, 接收机的性能也在逐渐 改善, 体积和重量在逐渐缩小, 这在一定程度上降低了测量 工作的难度。目前的 GPS 接收机已经逐渐向着渐变画、小型 化的方向发展, 测量人员在使用过程中, 只需通过天线整平、 高度量取以及电源开闭, 即可完成信息数据的自动化收集和 处理工作。

\section{GPS 技术在工程测量中的应用}

3.1 工程测量中的应用

GPS 定位技术中包含的专业学科知识相对较多, 如几何 图形知识、物理学知识等, 通过这些理论知识的应用为工程 测量工作的开展提供了有利保障。工程测量中的定位技术在 应用中需要设置地面测量信号, 通过对地面测量信号中数据 的接收和传送, 来准确掌握被测对象的信息。工程测量中的 定位技术主要分为静态定位技术和动态定位技术两种。

静态定位技术在使用过程中, 先进行接收装置的安装, 并合理控制装置间的距离, 之后再通过较长时间的数据观测 来获取准确的测量信息, 接下来工作人员会对这些信息数据 予以分析和处理, 找出其存在的差异性, 为后续作业的开展 提供帮助。而应用动态定位技术, 则主要以地载波观测测量 为主, 通过控制基站的安装来实现信号接受和处理, 为人们 提供大量的动态信息数据。两者相比, 前者的操作要更加简 便, 且信息数据获取较为稳定, 后者因受到动态性特征的影 
响, 在信息分析上存在一定的难度。

3.2 工程水准点测量中的应用

传统测量技术在应用过程中, 很难对工程水准点完成准 确的观测, 并及时做好实地考察工作, 其很容易在观测中存 在误差, 影响实际测量效果。但是在应用 GPS 技术后, 通过与 卫星信号的实时连接, 提升了水准点定位测量的准确性和及 时性。实际操作原理为: 在水准点测量前, 要先实行天线设 施和信号接收机的安装, 以便于测量中能够及时、快速的接 收到微信信号, 之后实行卫星连接, 开始测量工作, 在测量过 程中, 要及时对观测数据予以记录, 了解数据的变化特征, 得 到较为精准的水准点测量数据, 为后续项目的开展提供依 据。通过 GPS 技术的应用, 大大提高了水准点测量工作的效 率和质量。

\subsection{GPS 虚拟现实技术在工程测量中的应用}

传统工程测量中需要耗费较大的人力、物力, 且很容易 受到环境因素的制约, 使测量结果的准确性无法得到保障, 严重时还会阻碍工程测量工作的开展。而利用 GPS 虚拟现实 技术后, 能够很好的解决传统测量中存在的各类问题, 实现 数据信息之间的交互和存管, 保证测量工作的质量和安全。 GPS 虚拟现实技术是利用计算机系统, 在软件中构建工程测 量具体模型, 然后通过三维形式展现出来。在测绘的过程中, 人员无需到达施工环境中进行测量施工模型的测绘, 通过虚 拟现实技术, 便可以明确测绘中的重点, 并有效处理其在测 量中的安全事项。

3.4 GPS 与传统技术融合在工程测量中的应用

同传统测量技术相比, GPS 技术在测量准确性上、操作 便捷性上均有利显著的改善, 不过受到积水水平的限制, GPS 在使用中还存在一些不足之处。尤其是在卫星信号较弱、存 在较多遮蔽物的区域内, 很难有效发挥出 GPS 技术的作用和 价值, 进而阻碍了测量工作的开展。为此需要将 GPS 技术与 传统技术进行有效结合, 促进测量工作的有序开展。现阶段, 最常用的融合方式就是将传统的解析法以及图解法与 GPS 技术实行有效融合, 以此来防止信号薄弱导致的观测数据不 准确现象的发生, 增大测量的精确度, 减少区域条件限制对
测量工作的影响。

3.5 在变形监测中的应用

在建筑工程项目开展中, 因为材料质量、承载能力等因 素的影响使得建筑存在不同程度的变形, 导致建筑质量出现 问题。为此在工程建设中, 需要开展合理的变形监测工作, 及时掌握建筑结构存在的变形情况, 然后制定合理的解决措 施, 提高建筑工程的质量。建筑物变形监测主要包括建筑物 沉降监测、建筑物倾斜监测、建筑物裂缝监测、建筑物位移 监测等。因为传统的变形监测存在一定的不稳性和不全面性, 所以建筑内存在一定的安全隐患, 增大了建筑的使用风险。 但是采用 GPS 技术后，由于其具有操作方便、观测时间快、 精度高、自动化、测站之间无需通视等优点, 能够很好的满 足变形监测的要求, 从而加强了检测数据的准确性和精度, 减少成本费用的支出, 因此被广泛应用在变形监测之中。

\section{4 结语}

综上所述, GPS 技术在工程测量中的应用, 能够及时准确 的完成被测对象的定位, 快速获取被测对象的信息数据, 从 而提高工程测量的效率, 保证测量数据的准确性, 为我国建 筑行业的发展创造有利条件。

\section{[参考文献]}

[1]罗毅.GPS 测量技术在工程测量中的应用 [ J].工程技 术研究,2017,(2):37。

[2]刘帅,孙付平, 应文䒨, 等.两种软件在 GPS 动态测量数 据后处理中的比较与分析[J].测绘工程,2014,23(03):66-68.

[3] 潘素萍.工程测量 GPS 动态监测应用与数据处理分析 [J].中国高新技术企业,2014,(02):43-44.

[4]武晓龙, 许斌锋, 徐爱霞, 等.应用 GPS 测量技术建构物 动态监测思路探讨 [J].科技资讯,2010,(07):64+66.

[5]周强.探讨工程测量 GPS 测量技术应用[J].门窗,2018,(3):231.

[6]陈玉名.浅析 GPS 测量技术在工程测量中的应用 [J]. 民营科技,2018,(06):34.

[7]李明.工程测量中 GPS 测量技术的实际应用[J].中国 高新区,2017,(24):30. 